\title{
Ética en revistas científicas
}

\author{
Ethics in scientific journals
}

\author{
Tomàs BAIGET \\ El Profesional de la Información, EPI SCP, Gran Vía Corts Catalanes 440, \\ 08015 Barcelona, baiget@sarenet.es
}

\begin{abstract}
Resumen
Revisión de malas prácticas y comportamientos fraudulentos en revistas científicas. Se realiza un análisis de la bibliografía sobre el tema, y se aportan experiencias personales derivadas de la participación en comités de evaluación y de redacción. La gran competitividad que existe en la sociedad propicia que autores, revisores y editores sientan la tentación de cometer infracciones que pueden distorsionar el verdadero conocimiento y erosionar la confianza establecida en el actual sistema científico. Las faltas pueden ser de diferente nivel de gravedad, desde fabricación de pruebas, falsedad y plagio, hasta una variada picaresca para conseguir más con menos esfuerzo. La mayor transparencia conseguida gracias a la mayor accesibilidad y conocimiento tanto de los artículos como de sus autores ayuda a mejorar la calidad y honestidad de la publicación científica.
\end{abstract}

Palabras clave: Ética. Malas prácticas. Fraudes. Revistas científicas. Entorno académico. Publicación científica.

\section{Introducción}

La humanidad va evolucionando hacia la perfección. Decir eso es una aseveración muy temeraria, pero en el fondo lo queremos creer fervientemente. Para fundamentar empíricamente esto deberíamos disponer de unas estadísticas de delitos/habitante a lo largo de los siglos, incluso pudiendo asignar un peso a cada delito. Imposible.

Pensar que tan sólo hace 70 años se produjo la aberración del nazismo en una sociedad tan avanzada como la alemana es algo bastante frustrante como para mantener la teoría de que mejoramos.

Sin embargo, se puede observar que, al menos en algunas parcelas, o en algunas sociedades, sí se mejora. Las legislaciones intentan ajustarse e ir afinando la organización de la sociedad, dejando menos lagunas y cabos sueltos para evitar los delitos. Quizá no sea por la máxima de la convivencia de "vive y deja vivir", quizá no sea porque nuestras mentes se vuelven más respetuosas con el prójimo, sino que lo que ocurre es que la ley se hace más disuasoria y va resultando más peligroso delinquir.

\begin{abstract}
The literature on malpractice and misconduct in scientific journals is reviewed and personal experiences derived from the participation of the author in evaluation and editorial committees are discussed. The great competitiveness of the society today causes authors, reviewers and editors to be more tempted to commit offenses that may distort the truth and erode the trust established in the current system of reporting science. These can be of different severity, from fabrication of evidence, falsification and plagiarism to picaresque attempts to get more credit with less effort. Improved transparency through increased access to scientific papers and information about their authors helps to improve the quality and integrity of scientific publishing.
\end{abstract}

Keywords: Ethics. Malpractice. Frauds. Scientific journals. Academic environment. Scientific publications.

Está claro que esto está sucediendo en la actualidad, aunque no sabemos hasta qué punto los sistemas judiciales y policiales pueden llegar a controlarnos más de lo que deseamos las personas "normales" en el futuro. Pero eso no es nuestro tema.

La parcela de la publicación científica es una de las que, en conjunto, está evolucionando a mejor. Hunter (2000) dice:

Las normas éticas siguen creciendo. Muchas de esas pautas se hubieran considerado excesivas tan solo hace 10 años, no porque no tuvieran consecuencias prácticas, sino porque muchos investigadores no consideraban que la publicación duplicada, por ejemplo, fuera inaceptable. Pero las actitudes han cambiado significativamente, y a mejor, porque se ha pensado y se ha debatido mucho sobre estos temas.

Las múltiples redes de contactos creadas en el campo científico han introducido mucha luz y mucha transparencia, y por otro lado cada vez es más difícil transgredir las normas impunemente.

En el entorno de las revistas se van introduciendo mejoras de funcionamiento e incluso se van profesionalizando siguiendo pautas como las 
que publicó Delgado (2001). La profesionalización es buena porque permite exigir buenos resultados, al contrario que el trabajo de aficionado, realizado en horas libres, que siempre hay que tomarlo con benevolencia, como una aportación de buena voluntad. Y la creación de un entorno de trabajo más serio y ordenado favorece las buenas conductas.

Pero ¿cuáles son los "delitos" y "faltas" cometidos todavía en este ámbito de las revistas científicas? En este artículo nos hemos propuesto hacer una revisión de los mismos, así como analizar algunos mecanismos para contrarrestarlos.

\section{Necesidad de las normas}

La publicación científica ha entrado en una situación de ser un juego con una feroz competición: los jugadores luchan para acumular puntos a partir de sus publicaciones en revistas arbitradas, a veces y por desgracia contando más en términos de números que de calidad. Las publicaciones son importantes para la promoción universitaria, para apoyar la credibilidad del solicitante al pedir una subvención, contribuir al nombramiento en un comité influyente, $y$, en el sentido más amplio son la materia sobre la cual se construye la fama y la fortuna, y se pueden ganar brillantes premios. Pero los juegos competitivos requieren normas acordadas y árbitros con autoridad para hacer cumplir dichas normas (Edwards, 1995).

El incumplimiento de las normas éticas - ya sea debido a ignorancia, al engaño intencional inducido por un deseo de obtener ganancias comerciales o avances académicos, o por la indiferencia mostrada ante la importancia de la ética en la publicación - corroe nuestra confianza en la ciencia y en la sociedad. En consecuencia, los gobiernos han decidido que estemos expuestos al escrutinio cada vez mayor de los medios de comunicación y de sus agencias, convencidos de se requieren mejoras en este sistema autorregulado. Las revistas, por su parte, están ahora mucho más dispuestas a hacer cumplir las normas éticas (Roberts, 2009).

Las personas necesitamos que de vez en cuando se nos recuerden las normas y que esto no es la selva, ni tan sólo Jauja, como para hacer lo que nos parezca. Por ello, estamos convencidos de que sólo por el hecho de sacar el tema aquí vamos a contribuir a mejorar un poco la calidad y la equidad de la publicación científica.

En años recientes se han ido creando comités y consejos nacionales e internacionales, que asesoran sobre las mejores prácticas. Como casi siempre ha ocurrido en documentación, los médicos han sido los adelantados:

En 1997, Richard Smith, editor jefe del British Medical Journal, fundó el Committee on Publication Ethics (Cope). El Cope ha publicado las Best Practice Guidelines for Journal Editors y posteriormente el Code of conduct, cuyas últimas versiones pueden descargarse gratuitamente de su web (ver referencias al final).

En junio de 2008 se presentó la Equator Network (Enhancing the Quality and Transparency of Health Research), que había iniciado sus trabajos en 2006. En su web dispone de una serie de guías dirigidas a autores, revisores y editores, especialmente de revistas médicas. En una primera etapa trata de mejorar la comunicación (reporting) pero en los próximos años se propone asesorar e intervenir también en la calidad de la investigación.

Otros organismos, asociaciones y revistas han publicado documentos para publicar, que incluyen normas, recomendaciones, buenas prácticas, pautas éticas, etc., entre ellos:

- International Committee of Medical Journal Editors (ICMJE), que publica los Uniform Requirements for Manuscripts Submitted to Biomedical Journals.

- World Association of Medical Editors (WAME). http://www.wame.org/

- Office of Research Integrity (ORI), US Public Health Service. http://ori.dhhs.gov/

- American Psychological Association (APA). Working Group on Journal Article Reporting Standards (JARS Group). http://www.apa. org/pubs/authors/jars.pdf.

- Council of Science Editors (CSE). White Paper on Promoting Integrity in Scientific Journal Publications. http://www.councilscience editors.org.

- PLoS ONE Editorial and Publishing Policies. http://www.plosone.org/static/policies.action.

\section{Condiciones que llevan a infringir}

Según Breen (2003) y Redmang; Merz (2005) las condiciones profesionales que pueden potenciar la mala conducta científica son: la presión del "publicar o perecer", requisitos para la financiación de la investigación, falta de integridad intelectual, ignorancia de la ética de la ciencia, afán de promoción profesional, arrogancia...

También puede haber causas personales como: fama, falta de conciencia moral, ganancias financieras, deshonestidad, incompetencia, pereza, enfermedad mental... 
Sin olvidar las causas sociales: estructura de recompensas inadecuada, ambiente de trabajo caótico, falta de supervisión, carga de trabajo excesiva...

Cuando se infringen las normas se acostumbra a pensar que los perjudicados son otros autores, revistas, instituciones $y$, por supuesto, la Ciencia en abstracto, pero es interesante esta consideración del engaño que se hace al lector que consta en los "Uniform requirements..." (ICMJE, 2009):

Los lectores de revistas fuente merecen poder confiar en que lo que están leyendo es original, a menos que se mencione claramente que el artículo está siendo re-publicado por haberlo convenido autor y editor. La base de esta posición son las leyes internacionales de copyright, la conducta ética, y un uso eficiente de los recursos.

Los lectores pueden sentirse estafados también si pagan dos veces por un mismo contenido.

\section{Tipos de infracciones}

En general la conducta científica fraudulenta (scientific misconduct) corresponde a las siglas FFP —fabricación, falsificación y plagio-, aunque algunos comités y organismos añaden "engaño y desviaciones deliberadas, peligrosas 0 negligentes". La diferencia entre "fabricación" y "falsificación" no está muy clara, el primer término parece referirse más bien a fabricación de pruebas o a elaboración artificial de algo sin seguir el método explicado en el artículo; y el segundo parece que se refiere a cambiar los datos obtenidos para aparentar la confirmación de una hipótesis.

La definición legal de "mala conducta científica" a efectos de imponer sanciones ha llevado alguna controversia. Una fecha citada con frecuencia en la bibliografía es la del 16 de abril de 2004, cuando el US Public Health Service (PHS) publicó una revisión de las normas y cambió la definición (v. Department of Health and Human Services, 2004). La nueva norma define la mala conducta de investigación como FFP, omitiendo la frase que antes existía "y otras prácticas que se apartan seriamente de las aceptadas en la comunidad científica". Tal frase había sido criticada por su ambigüedad y por su mal uso al sancionar actos de mala conducta profesional o académica (Schachman, 1993).

Antes de entrar más en materia vamos a delimitar este estudio a lo que es estrictamente publicación en revistas. Así, aquí no trataremos de las infracciones producidas durante los proyectos de investigación como, por ejemplo:
- no cumplir la normativa legal sobre ensayos con personas y animales;

- no asegurar la confidencialidad de datos de pacientes;

- falsificar datos y resultados (Delgado et al., 2007);

Veamos, pues, las malas conductas producidas en el proceso editorial de una revista. Una posible clasificación, que seguiremos, sería según quien las comete: autores, editores y evaluadores.

\section{5. Ética de los autores}

Los autores están siendo sometidos a una enorme presión para que publiquen, pues en ello les va no sólo su reputación sino su sueldo. Esto es un sistema que quizá sea bueno en su conjunto, pues así se tiene interés en dar a conocer los resultados de la investigación, pero también da lugar a que se produzcan bastantes de los fallos del sistema de la publicación científica:

\section{Plagio}

Es sin duda la falta más grave que puede cometer un autor. Según leemos en cualquier diccionario, plagiar es robar y hacer pasar como propias las ideas o palabras de otros; usar una obra sin acreditar la fuente; o presentar como nuevo $u$ original un producto o una idea ya existente. Trataremos el plagio más adelante.

2. Duplicidad total o parcial de versiones de los mismos resultados de una investigación.

Entre las estratagemas usadas para conseguir publicar varias veces lo mismo están:

- cambiar el título y el resumen;

- mantener la parte teórica y cambiar la aplicación práctica;

- añadir autores amigos aunque no hayan participado, sólo para disfrazar el artículo;

- traducir a otro idioma;

- publicar artículos con menos del 50\% de novedad.

La publicación duplicada es un auto-plagio, tan detestable como copiar de otros (Serebnick y Harter, 1990).

Presentar un original simultáneamente a dos revistas es una ingenua mala práctica debida a la ignorancia, que cada vez es menos frecuente. Si el trabajo fuera aceptado en ambas implicaría para el autor pasar a figurar en una lista negra que le impediría publicar en esas revistas, y quizá también en otras, pues para los editores muchos de los cuales se conocen y colaboran eso es una falta muy grave. 
Tampoco es aceptable presentar como artículo la comunicación presentada a un congreso que se ha publicado en las actas del mismo, aunque éstas tengan poca difusión. Si por la importancia del trabajo autor y editor acuerdan hacerlo, ello debe hacerse constar claramente.

Otra práctica que ha perdurado hasta hace pocos años, por mentalidad todavía de la época del papel, y hoy ya en desuso, es la publicación en una revista de un trabajo que ya figura en una web o un cd-rom, por ejemplo formando parte de las actas de un congreso que sólo se han publicado en versión electrónica. Actualmente eso no es admisible.

En inglés se denomina watering down research (diluir la investigación) la práctica de fragmentar artificialmente una investigación en unidades publicables mínimas, lo que se conoce también como publicación salami. No puede tacharse de mala conducta, pero es evidente que esa dispersión de resultados no es buena para la ciencia.

Entre otras prácticas cuestionables, Bebeau y Davis (1996) listan:

- citar fuentes sin haberlas leído;

- no corregir interpretaciones erróneas de datos observadas en artículos de otros;

- omitir datos que contradicen una investigación propia anterior;

- liberar resultados científicos prematuramente, antes de su revisión por expertos;

- mantener durante varios años en secreto las conclusiones de una investigación;

- no compartir datos y métodos que permitirían a otros re-examinar o replicar los experimentos, o realizar nuevas investigaciones;

- no guardar los datos primarios de una investigación;

- acreditar autorías falsas o basadas en criterios distintos de la aportación al trabajo.

Sobre esto último, los autores deben firmar los artículos según su contribución a los mismos, sin falsear autorías, ni en más ni en menos, responsabilizándose de los resultados.

A la anterior lista se puede añadir:

- poner un título engañoso;

- enunciar algo en el resumen que luego no se trata correcta o suficientemente en el texto;

- no declarar que el contenido de un artículo procede de una tesis doctoral;
- intercambiar citas entre colegas amigos sin que hayan participado en el estudio ni en el artículo.

\section{Sobre las reseñas}

Las reseñas y críticas de libros deberían ser tan rigurosas como las evaluaciones de artículos, pero el hecho de hacerlas firmando con nombres y apellidos hace que casi siempre se conviertan en panegíricos de alabanza al autor del libro. Al final resulta que todos los libros "son imprescindibles, hacían mucha falta y son de compra obligada". Pero si un libro es muy malo entonces la reseña no se publica. Para evitar esta comedia algunas revistas han decidido no publicar reseñas de libros.

Algo parecido ocurre con las reseñas de congresos: quien las firma se ve obligado a hablar de forma "equilibrada" de todos los ponentes, buenos y malos. Nadie ha escrito nunca, al menos que sepamos en España: "Una vez más, Fulano ha contado el mismo rollo de siempre", y sin embargo cuántas veces lo habremos pensado. Incluso no hay nadie que quiera firmar una reseña en la que simplemente se deje de mencionar al tal Fulano, pues "se podría molestar" si no se habla de él. En cambio se acepta que Fulano sea un desconsiderado con toda la audiencia, haciéndole perder el tiempo con una presentación repetida año tras año.

Esta falsa educación produce reseñas de congresos muy pobres que con el escaso espacio disponible en las revistas se convierten en poco más que una reproducción del programa, en vez de dedicarse a exponer las tres o cuatro cosas nuevas e interesantes escuchadas en las sesiones.

\section{7. Ética de los editores}

El problema de los editores es encontrar buenos artículos, innovadores e interesantes, para publicar. A medida que la revista sea más conocida y esté indizada en las grandes bases de datos la facilidad de recibir buenos artículos aumenta, hasta el punto de que el problema puede llegar a ser cómo seleccionar y desechar el exceso, los menos buenos.

Si la revista consigue ser indizada por Scopus (de Elsevier) o Science Citation Index (de Thomson Reuters), su preocupación pasa a ser cómo aumentar su SJR (Scimago journal rank) o su FI (Factor de impacto) para destacar frente a las otras revistas de la misma temática. Ahí podemos encontrar una de las argucias de algunos editores: intentar añadir citas a artículos publicados en su revista a la bibliografía citada en los artículos recibidos. Las citas a trabajos publicados en la propia revista (autocitas, en la 
terminología de la bibliometría) computan en el cálculo de los citados índices.

La verdad es que la citación acostumbra a ser un área flexible y difusa, por lo muchísimo que se podría citar en cada bibliografía. Actualmente casi ningún autor es capaz de leer todos los escritos sobre su tema que han precedido al suyo - y probablemente en el apartado de bibliografía tampoco cabría-, por lo que normalmente aceptará la eventual sugerencia del editor de incluir la cita de algún artículo relevante publicado anteriormente en la revista.

De hecho cualquier autor avisado y educado antes de enviar un artículo a una revista procurará leer todo lo que se haya podido publicar en la misma sobre su tema, y lo citará si lo encuentra apropiado. También se da el caso de que el autor quiera congraciarse con la revista, en la creencia de que tanto editor como evaluadores le mirarán con mejores ojos para aprobar la publicación del artículo, y cite artículos de la revista que ni tan solo ha leído.

Cada vez más, las revistas serias no aceptan secciones de "bibliografía" sino de "bibliografía citada" o "referencias", es decir, la Redacción borra las citas que no son tales, que sólo son de relleno. Y de acuerdo con esta política los editores de esas revistas no van a intentar colar autocitas que el autor no haya usado verdaderamente.

La publicidad es una importante fuente de ingresos para muchas revistas comerciales. Sin ella algunas no podrían subsistir o deberían incrementar el precio de las suscripciones. Los editores pueden estar tentados a aceptar artículos que tengan sesgos favorables a los productos de los anunciantes.

Generalmente las reseñas de bases de datos y softwares comerciales originan serias discusiones técnicas entre autor y vendedor, que son muy positivas para acordar puntos de equilibrio justos y honestos sobre las características. Los sistemas son complejos y su rendimiento depende de muchos factores: capacidad de la máquina donde están instalados, módulos adquiridos, conexiones de datos, configuración o parametrización, etc. $\mathrm{Ni}$ autor ni editor deben ceder ante las presiones de los vendedores si no ven clara alguna función o contenido, aunque "peligre" la venta de publicidad.

\section{8. Ética de los evaluadores}

Los evaluadores son académicos o profesionales senior que se avienen a revisar de forma crítica y constructiva los manuscritos de los autores cuando el editor se lo pide. Es la llama- da "evaluación por pares" o "peer review" (peer = par o persona del mismo nivel de conocimientos). En la gran mayoría de revistas los revisores o pares no cobran, y sus únicas compensaciones son:

- conocer novedades antes que nadie;

- aparecer como evaluador en los créditos de la revista, sea formando parte de un Comité Científico Asesor fijo, y/o en una lista de evaluadores que cada año o cada dos publican preceptivamente las revistas.

Por nuestra experiencia en varias revistas, los evaluadores realizan de forma anónima y altruista un magnífico trabajo de mejora de los originales. A menudo dedican horas de su propio descanso a realizar minuciosas sugerencias para que un autor mejore su artículo. En las revistas que funcionan en sistema "doble-ciego", en el que autores y evaluadores no se conocen, los autores nunca sabrán quien les revisó su artículo. Evidentemente el evaluador sí sabe quien es el autor en cuanto se publica el artículo.

Que este sistema funcione es una de las cosas bellas que tiene la ciencia.

Se puede afirmar, pues, que el sistema de revisión por pares funciona, en principio, bastante bien, aunque con frecuencia se produzcan problemas de homogeneización y disparidades de criterios.

La mayoría de faltas de ética que se producen en el proceso de evaluación son leves y se deben a la falta de recursos y de tiempo: la revista no puede permitirse un elenco de evaluadores a los que poder exigir rapidez y completud de revisión por un pago de dinero sustancial, o porque sea muy prestigioso colaborar con la revista.

Los evaluadores son los que son, con su escasez de tiempo y a veces de conocimientos, y quizá no haya posibilidad de ninguna alternativa.

Podríamos decir que si un evaluador no va a dedicar suficiente esfuerzo a cada evaluación, no debería ofrecerse para esta tarea, pero entonces quizá la revista se quede sin evaluadores.

Como clara falta de ética se puede dar el caso de que un revisor recomiende el rechazo de un paper porque los hallazgos contradicen el trabajo propio.

\section{Conflictos de intereses}

Un conflicto de intereses es un hecho conocido por cualquiera de los participantes en el proceso de publicación que, si se revela más tarde, puede 
hacer que algún lector se sienta razonablemente inducido a error o engaño (o un autor, revisor o editor se ponga a la defensiva). Los conflictos de intereses pueden influir en el juicio de los autores, revisores y editores y a menudo no se observan a primera vista (Callaham, 2003).

Los conflictos pueden ser personales, comerciales, políticos, académicos o económicos. Éstos pueden estar relacionados con el empleo, la financiación de la investigación, acciones de la compañía, pagos por conferencias, viajes, consultorías, o cualquier otro tipo de interés en la compañía que aporte algún tipo de beneficio al autor.

Todos estos intereses (o su ausencia) debe ser declarados por escrito por los autores a la presentación del manuscrito, y si el editor lo considera pertinente se publicarán con el artículo.

Los revisores, editores y miembros del consejo de redacción también pueden tener conflictos de intereses, y están obligados a declararlos anualmente. Los editores y revisores asignados a la revisión de un manuscrito concreto sobre el que pueden tener un conflicto de interés deben recusarse a sí mismos de la supervisión, y el manuscrito ser reasignado a otro evaluador que no tenga ese problema.

Los originales publicados por los miembros del consejo de redacción presentan un caso especial de potencial conflicto de intereses. La revisión de estos manuscritos será siempre supervisada por un editor de alto nivel (es decir, editor adjunto o superior) que revisará la decisión del editor asignado para tener la máxima objetividad antes de la decisión definitiva (Callaham, 2003).

\section{Plagio en España}

Ningún país se ha escapado de la lacra del plagio, cuya práctica acostumbra a estar en proporción inversa a su nivel cultural. En España ha habido muchos casos conocidos, incluso entre los padres y madres de la documentación, pero tenemos que pensar que fueron episodios de esas personas, hoy ya abuelas o desaparecidas, y propios de épocas ya superadas.

Como muestra sólo dos casos famosos: el denunciado en la revista Information world en español, actualmente EPI (Baiget, 1994). Un individuo tradujo (con errores) un artículo de una revista canadiense, cambió el título y lo firmó, presentándolo como ponencia en una conferencia celebrada en Toledo.

Y otro el de la ex-profesora de la Facultad de Biblioteconomía y Documentación de la Universidad de Barcelona Núria Amat, que plagió grandes extensiones de texto - del orden del $80 \%$ - en todos sus libros. En el último, La documentación y sus tecnologías, Ediciones Pirámide, fue denunciada ante los tribunales por tres de los autores plagiados, los cuales ganaron el juicio pasados 6 años (!): la condena a Amat fue irrisoria: retirar el libro de las librerías si es que quedaba alguno después de los seis años- y a pagar una indemnización simbólica (Abadal, 2002). Lo peor de todo es que ella seguía alardeando de sus proezas "porque los demás no saben difundir los conocimientos".

La postura de nuestra comunidad profesional ha sido siempre muy blanda respecto de los casos de plagio, ya sea por no considerarlo grave, por pereza o comodidad de no enfrentarse con nadie, o por miedos diversos.

\section{Lucha contra el plagio}

En años recientes se han puesto en marcha varias plataformas que permiten detectar la similitud de escritos en Internet. Algunos de ellos son:

- TurnITin: http://turnitin.com/

- Compilatio: https://www.compilatio.net/

- CopyScape: http://www.copyscape.com/

- FairShare: https://fairshare.attributor.com/

- Approbo: http://approbo.citilab.eu/

Pero el sistema más importante en el campo científico para detectar plagios y publicaciones duplicadas es CrossCheck, puesto en marcha por CrossRef, la empresa que gestiona los DOls (digital object identifiers), con la cooperación de todas las grandes editoriales científicas. Elsevier, Kluwer, Taylor \& Francis, Emerald..., han volcado los artículos de todas sus revistas en una gran base de datos común, y mediante un software de la empresa iThenticate se puede saber en un instante el porcentaje de originalidad que tiene un manuscrito. Los editores de las revistas hacen la comprobación antes de enviar los artículos al proceso de peer review. http://www.crossref.org/crosscheck.html

Ante una falta de conducta de un autor los editores pueden realizar distintas acciones, dependiendo de la gravedad, desde vetar al autor para que no publique más en la revista, a avisar a colegas de otras revistas, o a informar a los jefes del autor.

\section{Conclusiones}

Aunque la ética no parezca un tema de moda, el hecho real es que en los últimos quince años se crearon varios comités para dictar normas y 
recomendaciones tendentes a mejorar tanto la investigación como el sistema de publicación científica. A su vez, en éste, se observa una gran aumento de competitividad lo que hace que todos sus componentes: autores, evaluadores y editores se esfuercen por mejorar.

Las mejoras en las revistas pueden empezar intentando cumplir más criterios formales, como los Latindex, y a usar sistemas electrónicos de gestión de los originales como OJS. La profesionalización y la tecnificación, que tanto simplifica, crean además un entorno de trabajo propicio para que también se lleven a cabo buenas prácticas y se cumplan las normas éticas.

La tentación de sacar más beneficios de los debidos justa y honestamente está presente en todo momento, y es necesario ir recordando los principios éticos para que el sistema de la ciencia siga manteniendo la actual base de confianza.

No parece que de momento nadie haya encontrado una alternativa a la forma de acreditación en vigor, cuya enorme presión con el "publica o perece" da lugar a serias distorsiones y picarescas en la difusión de los resultados de la investigación.

Además de la competitividad entre revistas, también se observa que la facilidad de acceder a todo lo publicado (controlando eventuales plagios) y de conocer a los autores colegas estén donde estén del planeta (por lo que ya no son tan impersonales y desconocidos), ha propiciado un mayor cumplimiento de las normas éticas.

\section{Referencias}

Abadal, Ernest. Sentencia de vulneración de derechos de autor. Mensaje enviado a IweTel el 22/03/2002. http://listserv.rediris.es/cgi-bin/wa?A0=IWETEL.

American Psychological Association (APA). Working Group on Journal Article Reporting Standards (JARS Group). Reporting standards for research in psychology: Why do we need them? What might they be? // American psychologist. 63:9 (2008) 839-851.

Baiget, Tomàs (1994). Increíble plagio. // Information world en español, oct. 28. 21.

Bebeau, Muriel J.; Davis, E. L. (1996). Survey of Ethical Issues in Dental Research. // J. dent. Res. 75 (1996) 845-855. DOI: 10.1177/00220345960750021901

Breen, Kerry J. (2003). Misconduct in medical research: whose responsibility? // Internal medicine journal. 33 (2003) 186-191.

Callaham, Michael L. (2003). Journal policy on ethics in scientific publication. // Annals of emergency medicine. 41:1 (2003) 82-89.

Committee on Publication Ethics. Best practice guidelines for journal editors. London: BMJ Publishing Group, http://publicationethics.org/files/u2/Best_Practice.pdfthic s.org/files/u2/Best_Practice.pdf.
Committee on Publication Ethics. Code of conduct. London: BMJ Publishing Group http://publicationethics.org /files/u2/New_Code.pdf

Council of Science Editors, Wheat Ridge, Co, USA. White paper on promoting integrity in scientific journal publications, 2009 Update. http://www.councilscienceeditors.org

Delgado López-Cózar, Emilio (2001). Las revistas españolas de ciencias de la documentación: productos manifiestamente mejorables. // El profesional de la información. 10:12 (dic.) 46-56.

Delgado-López-Cózar, Emilio; Torres-Salinas, Daniel; Roldán-López, Álvaro. El fraude en la ciencia: reflexiones a partir del caso Hwang. // El profesional de la información. 16:2 (marzo-abril 2007) 143-150. DOI: 10.3145/epi.2007.mar.07

Department of Health and Human Services (2004). Public Health Service Policies on Research Misconduct, // Federal Register. 69:20778-20803 section 93:103.

Edwards, Griffith et al. (1995). Playing fair: science, ethics and scientific journals (Editorial). // Addiction. 90 3-8.

Equator Network, http://www.equator-network.org/

Hunter, J. M. (2000). Ethics in publishing; are we practising to the highest possible standards? (Editorial I). // British journal of anaesthesia, 85:3 (Sept 2000) 341-343.

International Committee of Medical Journal Editors (ICMJE). Rev. 2009. Uniform Requirements for Manuscripts Submitted to Biomedical Journals. http://www.nlm.nih. gov/bsd/uniform_requirements.html.

Office of Research Integrity (ORI), US Public Health Service (PHS), http://ori.dhhs.gov/

Redman, Barbara K.; Merz, Jon F. (2005). Evaluating the oversight of scientific misconduct'. // Accountability in research. 12:3 157-162.

Roberts, Jason. An author's guide to publication ethics: a review of emerging standards in biomedical journals. /I Headache. 49 (2009) 578-589.

Schachman, Howard K. (1993). What Is misconduct in science? // Science. 261 (1993) 148-149.

Serebnick, Judith; Harter, Stephen P. (1990) Ethical practices in journal publishing: a study of library and information science periodicals. // Library quarterly. 60:2 91-119.

World Association of Medical Editors. http://www.wame.org/

Recibido: 04-07-2010.

Aceptado: 04-07-2010 
